\title{
Genotype profiles for the Costa Rican population at 7 PCR-based loci
}

\author{
Bernal Morera ${ }^{2,3 *}$, Ana Isabel Morales C. ${ }^{1 *} \&$ G. Jiménez-Arce ${ }^{3 *}$ \\ 1 Unidad de ADN, Sección de Bioquímica, Organismo de Investigación Judicial, Poder Judicial, Heredia, Costa Rica. \\ anagates@sol.racsa.co.cr 2 Escuela de Biología, Universidad de Costa Rica, rbt@cariari.ucr.ac.cr \\ 3 CIHATA, Universidad de Costa Rica, Costa Rica. gjimenez @ cariari.ucr.ac.cr \\ * The three coauthors contributed equally to this work.
}

Received 06-VIII-2003. Corrected 13-X-2003. Accepted 14-X-2003.

\begin{abstract}
Complete electronic DNA profiles of 2006 randomly selected Costa Ricans, typed for 7 PCR-based loci, are presented. Such data may prove valuable for anthropological and forensic studies of the Costa Rican population. Rev. Biol. Trop. 52(3): 713-715. Epub 2004 Dic 15.
\end{abstract}

Key words: Forensic, electronic communication, DNA profiles, PCR-based loci, Costa Rica.

Palabras clave: Ciencias Forenses, datos electrónicos, ADN, PCR, Costa Rica.

The international standards recommended among others by the International Society for Forensic Genetics (ISFG), require the existence of population databases supporting the biostatistical calculations. This applied to paternity investigations ant analysis of biological remains of criminological interest in case of not finding an exclusion (Olaisen et al.1997, Gómez and Carracedo 2000). Thus, a detailed knowledge of the genetic structure of the Costa Rican population, is of great importance because only little reference information is available, especially taking in account its unique hybrid origin of this population (Morera et al. 2001 a, 2003).

One of the most relevant and visionary aims of the Costa Rican Judiciary Branch during the last decade was the implementation of DNA technology with forensic purposes. This process started in 1995. Consequently, the results of population studies on genetic markers for typing biological material were formally available in the local context since 1997 (Morales et al. 1997). Such analyses supported the first applications of this technology into the
Costa Rican courtrooms (Morera and JiménezArce 1998, Morales et al. 2004). Nevertheless, they were only recently published (Morales et al. 2001, Morera et al. 2001b).

For practical restrictions, traditional publications only expose summary data on population studies. However, electronic communication enables the dissemination of complete DNA profile data to the scientific community. Such data may prove valuable for forensic applications and population genetic studies (Budowle and Moretti 1999).

This report, in its electronic format provides genotype profile data for 2006 randomly selected Costa Rican samples which were typed for the loci HLA-DQA1, LDLR, GYPA, HBGG, D7S8, Gc, and D1S80 (rdnaloci.txt). The details about materials and methods are published elsewhere (Morales et al. 2001, Morera et al. 2001b, Morales et al. 2004).

These data may serve as a useful base for future characterization of highly polymorphic autosomal short tandem repeat (STR) loci (Budowle et al. 2001), Y-linked polymorphisms and SNPs (single nucleotide 
polymorphisms) (Bosch et al. 2002, Jobling 2001) and mtDNA variability (Morera 2001/2002) for forensic purposes in Costa Rica, as done throughout the Americas (Budowle et al. 2001, Grattapaglia et al. 2001, Pagano et al. 2001, Cifuentes et al. 2002, Morales et al. 2002, Berardi et al. 2003, Chiurillo et al. 2003, Gonzalez-Andrade et al. 2003, Luna-Vazquez et al. 2003, Paredes et al. 2003, Perez et al. 2003) and Europe (Gusmao et al. 2000, Perez-Lezaun et al. 2000, Aler et al. 2001, Bosch et al. 2002).

\section{RESUMEN}

Se presenta una versión electrónica de los perfiles genéticos completos de 2006 individuos de Costa Rica seleccionados al azar, quienes fueron caracterizados para loci 6 basados en PCR. Tales datos podrían ser valiosos para estudios antropológicos y forenses de la población costarricense.

\section{REFERENCES}

Aler, M., A. Salas, P. Sanchez-Diz, E. Murcia, A. Carracedo \& M. Gisbert. 2001. Y-chromosome STR haplotypes from a Western Mediterranean population sample. Forensic Sci. Int. 119: 254-257.

Bosch, E., A.C. Lee, F. Calafell, E. Arroyo, P. Henneman, P. de Knijff \& M.A. Jobling. 2002. High resolution Y chromosome typing: 19 STRs amplified in three multiplex reactions. Forensic Sci. Int. 125: 42-51.

Berardi, G., U. Toscanini \& E. Raimondi. 2003. STR data for the PowerPlex 16 loci system from Buenos Aires population, Argentina. Forensic Sci. Int. 134: 222224.

Budowle, B., B. Shea, S. Niezgoda \& R. Chakraborty. 2001. CODIS STR loci data from 41 sample populations. J. Forensic Sci. 46: 453-489.

Budowle, B. \& T. R. Moretti. 1999. Genotype Profiles for Six Population Groups at the 13 CODIS Short Tandem Repeat Core Loci and Other PCRBBased Loci. Forensic Science Communications, Vol.1 (2). [Available on line at: http://www.fbi.gov/hq/lab/fsc/cu rrent/index.htm]

Chiurillo, M.A., A. Morales, A.M. Mendes, N. Lander, F. Tovar, A. Fuentes \& J.L. Ramirez. 2003. Genetic profiling of a central Venezuelan population using 15
STR markers that may be of forensic importance. Forensic Sci. Int. 136: 99-101.

Cifuentes, L., M. Acuna \& D. Sepulveda. 2002. Allele frequencies for six STR in a Chilean population. J. Forensic Sci. 47: 904-905.

Gómez, J. \& A. Carracedo. 2000. The 1998-1999 collaborative exercises and proficiency testing program on DNA typing of the spanish and portuguese working group of the international society for forensic genetics (GEP-ISFG). Forensic Sci. Int. 114: 21-30.

Gonzalez-Andrade, F., D. Sanchez \& B. Martinez-Jarreta. 2003. Genetic profile of the Ecuadorian mestizo population (Ecuador-South America) by using the PowerPlex 16 loci system kit. Forensic Sci. Int. 135: 64-66.

Grattapaglia, D., A.B. Schmidt, C. Costa e Silva, C. Stringher, A.P. Fernandes \& ME. Ferreira. 2001. Brazilian population database for the 13 STR loci of the AmpFISTR Profiler Plus and Cofiler multiplex kits. Forensic Sci. Int. 118: 91-94.

Gusmao, L., P. Sanchez-Diz, C. Alves, M.V. Lareu, A. Carracedo \& A. Amorim. 2000. Genetic diversity of nine STRs in two northwest Iberian populations: Galicia and northern Portugal. Int. J. Legal Med. 114: 109-113.

Jobling, M.A. 2001. Y-chromosomal SNP haplotype diversity in forensic analysis. Forensic Sci. Int. 118: 158-162.

Luna-Vazquez, A., G. Vilchis-Dorantes, L.A. PaezRiberos, F. Muñoz-Valle, A. Gonzalez-Martin \& H. Rangel-Villalobos. 2003. Population data of nine STRs of Mexican-Mestizos from Mexico City. Forensic Sci. Int. 136: 96-98.

Morales, J.A., J.C. Monterrosa, J.C. Alvarez, C. Entrala, J.A. Lorente, M. Lorente, B. Budowle \& E. Villanueva. 2002. Population data on nine STR loci in an El Salvadoran (Central American) sample population. J. Forensic Sci. 47: 900-901.

Morales C., A.I., B. Morera \& G. Jiménez-Arce. 1997. Proyecto Implantar en Costa Rica la detección de huellas genéticas humanas para la identificación individual en casos criminalisticos y de paternidad. Informe Final. [Reproduced In Morales et al. 2003. Rev. Latinoam. Der. Méd. y Med. Legal. 8(2): in press].

Morales-Cordero, A.I., B. Morera, G. Jiménez-Arce, G. Sánchez-Rivera, F. Calafell \& R. Barrantes. 2001. Allele frequencies of the markers LDLR, GYPA, HBGG, D7S8, Gc, HLA-DQA1, and D7S80 in the general and minority populations of Costa Rica. Forensic Sci. Int. 124: 1-4. 
Morales C., A.I., B. Morera \& G. Jiménez-Arce. 2004. La implementación forense de la tecnología del ADN en Costa Rica: Un análisis retrospectivo. Rev. Biol. Trop. 52: in press.

Morera, B. 2001/2002. Análisis del Polimorfismo del ADNmt en la Población General de Costa Rica: Un Asunto Pendiente. Rev. Latinoam. Der. Méd. y Med. Legal. 6/7: 21-34.

Morera, B. \& G. Jiménez-Arce. 1998. Identificación de restos óseos mediante análisis de ADN. Med. Legal Costa Rica. 15: 6-7.

Morera, B., R. Marín-Rojas \& R. Barrantes. 2001 a. Análisis de varios marcadores genéticos clásicos en la población de Costa Rica. Rev. Biol. Trop. 49: 1237-1252.

Morera, B., G. Sánchez-Rivera, G. Jiménez-Arce, F. Calafell \& A.I. Morales-Cordero. 2001b. Nicaraguan population data on LDLR, GYPA, HBGG, D7S8, Gc, and HLA-DQA1 loci. Rev. Biol. Trop. 49: 12531260.

Morera, B., R. Marín-Rojas \& R. Barrantes. 2003. Gene Admixture in the Costa Rican Population. Ann. Hum. Genet. 67: 71-80.
Olaisen, B., W. Bar, B. Brinkmann, B. Budowle, A. Carracedo, P. Gill, P. Lincoln, W.R. Mayr \& S. Rand. 1998. DNA recommendations 1997 of the International Society for Forensic Genetics. Vox Sang. 74: 61-63.

Pagano, S., J.C. Alvarez, C. Entrala, J.A. Lorente, M. Lorente, B. Budowle \& E. Villanueva. 2001. Uruguayan population data for eight STR loci (using the PowerPlex 1.2 kit). J. Forensic Sci. 46: 178.

Paredes, M., A. Galindo, M. Bernal, S. Avila, D. Andrade, C. Vergara, M. Rincon, R.E. Romero, M. Navarrete, M Cardenas, J. Ortega, D. Suarez, A. Cifuentes, A. Salas \& A. Carracedo. 2003. Analysis of the CODIS autosomal STR loci in four main Colombian regions. Forensic Sci. Int. 137: 67-73.

Perez, L., J. Hau, F. Izarra, O. Ochoa, U. Zubiate \& O. Garcia. 2003. Allele frequencies for the 13 CODIS STR loci in Peru. Forensic Sci. Int. 132: 164-165.

Perez-Lezaun, A., F. Calafell, J. Clarimon, E. Bosch, E. Mateu, L. Gusmao, A. Amorim, N. Benchemsi \& J. Bertranpetit. 2000. Allele frequencies of 13 short tandem repeats in population samples from the Iberian Peninsula and northern Africa. Int. J. Legal Med. 113: 208-214. 